\title{
The Canadian Head CT Rule; A Hospital Audit
}

\author{
John Kinyua \\ Coast General Hospital
}

Correspondence to: Dr John Kinyua, P. O BOX 90231 - 80100 Mombasa, Kenya. Email:johnkinyua@gmail.com

\begin{abstract}
Background: Minor head trauma is one of the leading cause of emergency department visits worldwide. The Canadian Head CT-scan rule (CCHR) in minor head injury is an evidence-based aid in decision making as regards to use of CT-scans to detect head injury requiring neuro-intervention. It therefore avoids wastage of resources. The objective was to compare the number of CT-scans done for minor head injury as compared to the number that would have been done if the CCHR was applied. Methods: A retrospective study was done. All patients resenting with minor head injury (GCS 13-15) were identified from the hospital registry and their files obtained. Patients not meeting the CCHR criteria excluded. Ten parameters were extracted and tabulated. Results: Forty-one patients were included with three exclusions. $89 \% \quad(n=34)$ of the patients presented with a 2 -hour GCS of 13 or more. $11 \%(n=4)$ were suspicious of base skull fractures. $23 \%(n=9)$ had signs of open fracture. Vomiting was seen in 2 patients $(5 \%)$.
\end{abstract}

\section{Introduction}

Mild traumatic brain injury is defined as a traumatically induced physiologic disruption of brain function as manifested by one of the following: any loss of consciousness, any memory loss, altered mental status at the time of the accident, focal neurological deficits which may or may not be transient (1). Operational criteria for clinical identification include: 1 or more of the following: confusion or disorientation, loss of consciousness for 30 minutes or less, post-traumatic amnesia for less than 24 hours, and/or other transient neurological abnormalities such as focal signs, seizure, and intracranial lesion not requiring surgery; Glasgow Coma Scale score of 13-15 after 30 minutes post-injury or later upon presentation for healthcare. These manifestations of minor traumatic brain injury must not
The mean age of patients was 29 years. 2 patients (5\%) reported amnesia. All the patients had a CT scan done. Fourteen patients would have required CT scans had the rule been used. Positive findings were noted in seven of the patients who qualified and in three who did not.

This demonstrated a $50 \%$ positive predictive value, a negative predictive value of $89 \%$, a sensitivity $70 \%$ and $75 \%$ specificity. Conclusion: Use of CCHR would reduce unnecessary use of CT scans in minor head injury in this setup.

Key words: Canadian, head, CT scan, rule, minor head injury

Ann Afr Surg. 2018;15(2):57-61

DOI:http://dx.doi.org/10.4314/aas.v15i2.5

(C) 2018 Author. This work is licensed under the

Creative Commons Attribution 4.0 International

License.

Conflicts of Interest: None

Funding: None

be due to drugs, alcohol, medications, caused by other injuries or treatment for other injuries (e.g. systemic injuries, facial injuries or intubation), caused by other problems (e.g. psychological trauma, language barrier or coexisting medical conditions) or caused by penetrating craniocerebral injury (1).The complexity of the definition underscores the difficulty which the physician in an emergency department faces in the management of patients presenting with mild traumatic brain injury.Reported worldwide incidence of traumatic brain incidence is 100 to 300 per 100,000 (2). This figure denotes the persons who present to medical facilities with traumatic head injury. It is estimated that more than 600 per 100,000 people who have sustained mild traumatic brain injury do not present to hospital (3). 
The estimated casualty department presentation is therefore as high as 900 per 100,000 people. Extrapolating these statistics to the hospital where the audit was carried out with a catchment area of 939,000 people (4), this presents a patient population of between 939 to 2817 annually based on international incidence rates. With such a patient burden, there is need for a system to rapidly assess the patient and accurately determine whether further investigations are warranted or not. As such there exists a substantial disagreement in regards to the indication of CT scan in minor head injury (5). Unwarranted imaging leads to unnecessary wastage of resources in regards to time and money while inadequate imaging results in missed injuries with attendant morbidity and litigation risk (5).

The Canadian CT head rule was developed as an aid to decision making in minor head injury (6). The Canadian CT head rule focuses on five high risk factors. The first factor is 2-hour GCS of less than 13 which indicates deteriorating neurological function. The next factor is a suspected open skull fracture or a base of skull fracture. This differs from an obvious fracture where the operator is able to identify an open skull fracture or base of skull fracture, a finding which obviates the need for a CT scan. The final high-risk factors are presence of vomiting which may indicate a rise in intracranial pressures (7) and patients older than 65 years. Patients older than 65 years have a risk for subdural hematoma formation in the absence of any clinical findings (8) and thus the indication for imaging. Intermediate risk factors are amnesia and a dangerous mechanism of injury. These factors when applied to study participants were shown to be sensitive for intracranial injury. The excluded factors namely obvious open fracture, oral anticoagulation, moderate head injury (GCS <13) and age less than 13 years preclude the application of the rule as these findings necessitate immediate imaging. By employing 5 high risk and 2 medium risk factors (Index 1)Steill at al were able to demonstrate an accurate predictability for need for neuroimaging with findings that necessitated neuro-intervention (9). The tool has a demonstrated sensitivity of $92 \%$ (10). Abdalla et al validated the Canadian head CT rule in a setup similar to the institution of the audit and were able to show a $73.8 \%$ reduction in the number of CT scans done for minor head injury (11) In the institution of the study, anecdotal evidence shows that decision making on need for imaging in minor traumatic brain injury is dependent on the clinical acumen of the evaluating physician without a standardized protocol. The objective of this study was to determine whether application of the Canadian head CT rule would reduce the number of CT scans done in patients with minor head injury without significantly increasing the number of missed injuries.

\section{Methods}

The study was a retrospective file audit. It covered the period from January 1st 2016 to 31st December 2016. Files were requisitioned from the records department of Aga Khan Hospital, Mombasa. All patients presenting with minor head injury (GCS 13-15) were identified from the hospital registry and their files obtained. Patients aged less than 16 years; patients with obvious skull fractures; those on oral anticoagulants, GCS less than 13 and non-trauma cases were excluded as these parameters necessitate the need for a CT scan and are excluded in the derivation of the Canadian head CT rule. Files with incomplete data were also excluded. Ten parameters were extracted i.e. GCS 2 hours after injury, whether an open skull fracture is suspected, any sign of base of skull fracture, episodes of vomiting, age, amnesia, mechanism of injury, whether a CT scan was done, CT scan findings and whether neurosurgical intervention was required. Dangerous mechanisms as outlined in the derivation of the Canadian head $\mathrm{Ct}$ rule are a pedestrian struck by a vehicle, vehicle occupant ejected and fall from a height of more than three feet.

Positive CT scan findings as per the Canadian head CT rule were defined as clinically important injury found on CT scan which ordinarily would require admission and neurological follow up (8). All brain injuries were ruled significant except the following: solitary contusions less than 5mm, localized subarachnoid bleeds of less than $4 \mathrm{~mm}$, isolated pneumocephalus and closed depressed fractures not through the inner table (12).The data collected was cross tabulated to show how many of the patients identified had CT scans done. This was compared to the number of patients who would have had CT scans done had the Canadian head 
CT rule for minor head injury been followed. Results of the CT scans were compared to establish whether the patients who would have qualified for a CT scan had any positive findings. Comparison was also done to establish whether any of the patients who did not qualify for a head CT scan had any positive findings.

Authorization from the hospital's ethics and review board was obtained before commencement of the study.

\section{Results}

The study found a total of forty-one patients for the study period. Three patients were excluded from the study due to incomplete data. Eighty-nine percent $(n=34)$ of the patients presented with a 2-hour GCS of 13 or more. Eleven percent $(n=4)$ were suspicious of base of skull fractures; $23 \%(n=9)$ had signs of open fracture and $5 \%$ of the patients $(n=2)$ reported vomiting. The mean age was 29 years with 3 patients above the age of 65 years. Two patients $(5 \%)$ reported amnesia. Sixty-six percent $(\mathrm{n}=25)$ of the patients were involved in motor vehicle collusions where they were restrained passenger in vehicles which were not extensively damaged while $26 \%(\mathrm{n}=10)$ fell from a height of between 0 to 3 feet. Three patients were involved in fist fights. None of the mechanisms of injury was construed to be dangerous. Overlap was noted for the patients with positive factors such as suspicion for both open skull fractures and base of skull fractures.

Table 1: Positive Tindings as per

The Canadian Head Ct Rule*

\begin{tabular}{|l|l|}
\hline Findings & $\begin{array}{l}\text { Number of } \\
\text { Patients (\%) }\end{array}$ \\
\hline GCS $<13$ & $4(11)$ \\
\hline $\begin{array}{l}\text { Suspected Base of Skull } \\
\text { Fracture }\end{array}$ & $4(11)$ \\
\hline Suspected Open Fracture & $9(23)$ \\
\hline Amnesia & $2(5)$ \\
\hline Vomiting & $2(5)$ \\
\hline Age $>65$ & $3(8)$ \\
\hline Dangerous Mechanism & 0 \\
\hline Total Positive Findings & $14(37)$ \\
\hline
\end{tabular}

GCS- Glasgow come scale

*several patients had overlapping factors

All the thirty-eight patients had a CT scan done. Had the Canadian CT scan rule been followed, only fourteen patients would have required $\mathrm{CT}$ scans. Positive findings were noted in seven of the patients who qualified for CT scans, 2 patients had subdural hematomas, one had a cerebral contusion and 4 had skull fractures. Positive findings were also noted in 3 of the patients who did not qualify for CT scans. These findings were a maxillary fracture, a raptured tympanum and a base of skull fracture.

With fourteen patients requiring CT scans according to the rule and seven of them having positive findings this denotes a positive predictive value of $50 \%$. Ten out of the total thirty-eight patients had positive findings on CT scans which shows a $70 \%$ sensitivity of the rule in our setup and a $75 \%$ specificity. Twenty-five out of twenty-eight patients were correctly ruled not to require CT scans showing a negative predictive value of $87.5 \%$.

\section{Discussion}

In developed countries, the use of CT scans in traumatic brain injuries is very common but the yield for intracranial injuries is relatively low at $0.7-3.7 \%(7,13)$. This represents a cost of 135-216 million dollars annually (7). The Canadian Head CT (Appendix A) rule was developed with this in mind (13). It standardizes the emergency management of patients with mild traumatic brain injury with improved utilization of resources. This study showed that there is an overuse of CT scans for minor head injury patients. Employment of a simple tool such as the Canadian head CT rule which can be taught to medical officers in an emergency department setting could reduce the use of CT scans by $63 \%$, a figure demonstrated in several other centers $(8,9,11)$. With an $87.5 \%$ negative predictive value the rule can safely be used to inform the decision on whether or not to perform a CT scan. Education on high risk and intermediate risk factors in head trauma has also been shown to improve patient evaluation (10).Reduced GCS after 2hours was shown to be the greatest indication for a scan followed by skull fractures. These findings were clearly documented in the files sampled which shows that clinicians are aware of the indicators of possible intracranial injury $(1,2)$. The rule therefore serves as a standardization tool for various providers in their line of work. A CT scan in the developing world cost approximately 100 US dollars (3), a $63 \%$ reduction in CT scans done for minor head 
injury patients who number $>600$ per 100,000 (6) demonstrates a significant saving in terms of resources which can then be channelled to other uses. The greatest risk posed by this tool is the possibility of failing to perform a CT scan in a patient who requires it. We demonstrated a sensitivity of $70 \%$ if the rule was applied. Similar studies have shown a $66.67 \%$ sensitivity (13) which is comparative to this study. The rule was shown to have a negative predictive value $87.5 \%$. This shows that the rule if used correctly can accurately guide the medical officer as to who does not require imaging, clinical acumen must therefore some into play as over reliance on the rule has been shown to result in significant missed diagnoses (14). The greatest limitation of the study was that it was performed in retrospect. This means that the service providers did not have the benefit of this tool or other standardized format of assessment to streamline their decision making. A CT scan may therefore have been the surest way of ensuring that all possible injuries were identified, a phenomenon documented by Bouida et al (15). A follow up prospective study therefore needs to be done to compare whether use of the tool actually reduced the number of $\mathrm{CT}$ scans done for minor head injury.

\section{Conclusion}

Adoption of the Canadian CT Head rule in this setup would provide a standardized tool for ordering of head $\mathrm{Ct}$ scans in minor head injury thus improving patient evaluation while avoiding missed clinically significant intracranial injuries.

\section{References}

1. Carroll LJ, Cassidy JD, Holm L. et al. Methodological Issues and Research Recommendations for Mild Traumatic Brain Injury: The WHO Collaborating Centre Task Force on Mild Traumatic Brain Injury. J Rehabil Med. 2004(43 Suppl.), 113-25.

2. Cassidy JD, Carroll LJ, Peloso PM, et al. Incidence, Risk Factors and Prevention of Mild Traumatic Brain Injury: Results of The WHO Collaborating Centre Task Force on Mild Traumatic Brain Injury. J. Rehabil. Med.2004 (43 Suppl.), 28-60
3. Dacey RG, Alves WM, Rimel RW, et al. Neurosurgical Complications after Apparently Minor Head Injury: Assessment of Risk in a Series of 610 Patients. J Neurosurg. 1986; 65: 302-10.

4. http://www.agakhanhospitals.org/mombasa/

5. Kenya Census 2009 (Page 7). Total Population by county Retrieved on 19 August 2014.

6. Setnik L, Bazarian, JJ The Characteristics of Patients Who Do Not Seek Medical Treatment for Traumatic Brain Injury. Brain Inj.2007 21 (1), 1-9

7. Nee PA, Hadfield JM, Yates DW et al; Significance of Vomiting after Head Injury. J Neurol Neurosurg Psychiatry. 1999; 66(4):470-3

8. Adhiyaman V, Asghar M, Ganeshram $\mathrm{KN}$ et al; Chronic Subdural Hematoma in the elderly. Postgrad Med J. 2002; 78(916):71-5

9. Taheri PA, Karamanoukian H, Gibbons K et al. Can Patients With Minor Head Injuries Be Safely Discharged Home? Arch Surg 1993; 128(3): 28992.

10. Stiell IG Wells GAVandemheen $\mathrm{K}$, et al. The Canadian CT Head Rule for Patients with Minor Head Injury. Lancet 2001;357(9266):1391-96.

11. Abdalla RO, Qureshi MM, Saidi $\mathrm{H}$, et al; Introduction of the Canadian CT Head Rule Reduces CT Scan Use in Minor Head Injury. The Ann Afr Surg.2015;12 (1): 19-21

12. Sharif-Alhoseini M, Khodadadi H, Chardoli M, et al Indications for Brain Computed Tomography Scan after Minor Head Injury. J Emerg Trauma Shock. 2011;4(4): 472-6.

13. Arab AF, Ahmed ME, Ahmed AE, et al ; Accuracy of Canadian CT Head Rule in Predicting Positive Findings on CT of The Head of Patients after Mild Head Injury an A Large Trauma Centre in Saudi Arabia. Neuroradiol J. 2015 28(6): 591-7

14. Papa L, Stiell IG, Clement CM et al; Performance of The Canadian CT head Rule and The New Orleans Criteria for Predicting Any Traumatic Intracranial Injury on CT in a United States level I Trauma Center. Acad Emerg Med 2012; 19(1):2-10

15. Bouida W, Marghli S, Souissi S, et al; Prediction Value of The Canadian CT Head Rule and The New Orleans Criteria For Positive Head CT Scan and Acute Neurosurgical Procedures in Minor Head 
Trauma: A Multicenter External Validation Study.

Ann Emerg Med.201361(5):521-7.

\section{Appendix}

\section{Canadian CT Head Rule}

CT head is only required for minor head injury patients

with any one of these findings:

High Risk (for Neurological Intervention)

1. GCS score $<15$ at 2 hrs after injury

2. Suspected open or depressed skull fracture

3. Any sign of basal skull fracture*

4. Vomiting $\geq 2$ episodes

5. Age $\geq 65$ years

Medium Risk (for Brain Injury on CT)

6. Amnesia before impact $\geq 30 \mathrm{~min}$

7. Dangerous mechanism ** (pedestrian, occupant ejected, fall from elevation)

Rule Not Applicable If:

- Non-trauma cases

- GCS $<13$

- Age $<16$ years

- Coumadin or bleeding disorder

- Obvious open skull fracture

*Signs of Basal Skull Fracture- hemotympanum, 'racoon' eyes, CSF otorrhea/rhinorrhea, Battle's sign

** Dangerous Mechanism

- Pedestrian struck by vehicle

- Occupant ejected from motor vehicle

- Fall from elevation $\geq 3$ feet or 5 stairs13) *Steill IG, et al. The Canadian CT Head Rule for Patients with Minor Head Injury. Lancet

2001; 357:1391-96. 